\title{
JOURNAL OF RHEOLOGY
}

Comparison of Flow Birefringence Data with a Numerical Simulation of the Hole Pressure

D. G. Baird, M. D. Read, and J. N. Reddy

Citation: Journal of Rheology (1978-present) 32, 621 (1988); doi: 10.1122/1.549983

View online: http://dx.doi.org/10.1122/1.549983

View Table of Contents: http://scitation.aip.org/content/sor/journal/jor2/32/6?ver=pdfcov

Published by the The Society of Rheology

\section{Re-register for Table of Content Alerts}


Copyright by the American Institute of Physics (AIP). Baird, DG; Read, MD; Reddy, JN. "comparison of flow birefringence data with a numerical-simulation of the hole pressure," J. Rheol. 32, 621 (1988); http://dx.doi.org/10.1122/1.549983

\title{
Comparison of Flow Birefringence Data with a Numerical Simulation of the Hole Pressure
}

\author{
D. G. BAIRD and M. D. READ* Department of Chemical \\ Engineering and Polymer Materials and Interfaces Laboratory \\ and J. N. REDDY Department of Engineering Science and \\ Mechanics, Virginia Polytechnic Institute \& State University, \\ Blacksburg, Virginia 24061-6496
}

\begin{abstract}
Synopsis
The penalty-Galerkin finite-element method is used to simulate the flow of a polystyrene melt over a rectangular slot placed perpendicular to the flow direction. The White-Metzner constitutive equation is used with a Carreau model viscosity function and a shear rate-dependent relaxation time defined so that the primary normal stress difference is exactly reproduced by the model in simple shear flow. Values of the stress field predicted by the simulation are compared with those obtained experimentally by means of flow birefringence. As observed by others, the limiting elasticity value as determined by the Weissenberg number (We) for convergence of the algorithm decreased with increased refinement of the mesh. However, good agreement is still found between predicted values of stress using the coarse mesh and those measured by means of flow birefringence. This work suggests that there may be an optimum mesh for a given flow and constitutive equation which will still give physically realistic results. The Weissenberg number for the melt used in the experimental study asymptotically approached a value of about 1.5 with increasing shear stress, suggesting that it may not be necessary to reach excessively high values of We for simulations involving some polymer melts.
\end{abstract}

\section{INTRODUCTION}

Although considerable progress has been made in the last several years in the simulation of viscoelastic fluid flows, there are still two major unresolved problems: (1) the breakdown of numerical algorithms for nearly any type of nonlinear constitutive equation at values of elasticity parameters where the predicted flow behavior is just starting to deviate from that predicted for in-

*Present address: Dow Chemical Co., Midland, Michigan.

(c) 1988 by The Society of Rheology, Inc. Published by John Wiley \& Sons, Inc. Journal of Rheology, 32(6), 621-638 (1988) CCC 0148-6055/88/060621-18\$04.00 
elastic models; (2) the lack of experimental data for fluids whose rheological properties are described by the constitutive equation used in the simulation with which to compare the predictions of the simulations. Due to this lack of sufficient experimental data, we cannot be sure whether the predicted streamlines and stress fields are physically correct even under conditions when the numerical algorithm is convergent.

As an example of the last point we cite the work of Davies and co-workers, ${ }^{1}$ who carried out a simulation of both planar and axisymmetric 4:1 contraction flows using three different constitutive equations. They observed that vortices were predicted for both an upper convected Maxwell model and an Oldroyd fourconstant model. However, as the finite-element or differences meshes were refined, the size of the vortex decreased. This prompted them to conclude that the vortices observed with the coarse meshes were numerical artifacts. Furthermore, they compared their results with the streak photographs of Walters and co-workers, ${ }^{2,3}$ who presented results for both a Boger fluid and a highly shear-thinning polyacrylamide solution. The Boger fluid exhibited no vortices in planar entry flow while the polyacrylamide solution did. In this case the rheological properties measured in shear flow of the Boger fluid are best described by the Maxwell and Oldroyd models, while the polyacrylamide solution properties are best described by the White-Metzner model. No vortices were predicted with the White-Metzner model either, but convergence of the numerical algorithm occurred at very low values of the Deborah number (De) where De $=\lambda \dot{\gamma}$ and $\lambda$ is the relaxation time and $\dot{\gamma}$ is the shear rate. In any event, the predictions of the simulation in the case of the White-Metzner model are just opposite to what is observed.

Another point of interest in Walters et al.'s work was that the highest limiting value of De was obtained for one of the meshes with an intermediate degree of refinement and with further refinement this value decreased. These results then raise the question of whether there is an optimum mesh size which will give the highest possible limit of De and still give physically acceptable predictions. To answer this question it will be necessary to have available flow visualization data on different polymeric fluids whose rheological properties can be accurately described at least for viscometric flow and hopefully for simple extensional flow. 
Another flow situation where there are some experimental data available is that of flow over a rectangular cavity placed perpendicular to the flow direction. ${ }^{4-6}$ This flow is associated with the hole pressure. ${ }^{7}$ The first simulations reported in the literature were concerned with predicting the ratio of the hole pressure $\left(P_{H}\right)$ to the primary normal stress difference $\left(N_{1}\right)$ as a function of the Reynolds number (Re) and De and comparing this to the theoretical values in the limit as both $\mathrm{De}$ and $\mathrm{Re}$ went to zero. ${ }^{8-10}$ Jackson and Finlayson ${ }^{11}$ carried this work further by using a corotational Maxwell model with empirically defined viscosity and relaxation time functions. They were able to fit the viscometric properties of the polystyrene solutions used by Baird ${ }^{12}$ exactly. Their predicted values of $P_{H} / N_{1}$ agreed well with those reported by Baird. ${ }^{12}$ They noted, however, that there were severe convergence difficulties, especially when the slot width to die height ratio became less than 0.5. Malkus and Bernstein ${ }^{19}$ used the integral constitutive equation of Curtiss and Bird $^{14}$ in their simulation of the hole pressure. Based on their calculations they were able to explain the validity of the Higashitani and Pritchard theory ${ }^{15}$ which relates $P_{H}$ to $N_{1}$. Although they did not compare their predictions directly with experimental data, they arrived independently at the same conclusions as Pike and Baird, ${ }^{16}$ who used flow birefringence to determine the stress field in the region of a slot placed perpendicular to the flow direction for a polystyrene melt. Malkus and Webster ${ }^{17}$ computed the ratio of $P_{H} / N_{1}$ for the Maxwell model using both the finite-element method and the finite-difference method. They compared their results with the predictions of the calculations for the second-order fluid and found good agreement. They also concluded that their calculations corroborated experimental evidence that $N_{1}$ can be predicted via $P_{H}$ measurements.

The purpose of the present report is twofold. First, we want to determine whether it is possible to simulate the stress field, measured by means of flow birefringence, for flow of a polymer melt over a rectangular slot placed perpendicular to the flow direction when the constitutive equation at least fits the viscometric properties of the melt used in the flow experiments. Second, we would like to investigate the effect of mesh refinement on not only the predictions of the stress field, but also on convergence of the numerical algorithm by comparison with the stresses measured by means of flow birefringence. This article will particularly con- 
centrate on two regions of the flow. One is along the centerline of the slot and the other is along the mouth of the slot.

\section{GOVERNING EQUATIONS}

The steady incompressible flow of viscoelastic fluids is governed by the following equations:

(i) the equation of motion

$$
\rho v \cdot \nabla v=-\nabla p-\nabla \cdot \mathbf{T}+\rho g,
$$

(ii) the continuity equation

$$
\nabla \cdot v=0
$$

(iii) an appropriate constitutive equation which relates $\mathbf{T}$ to $\mathbf{v}$. Here $\mathbf{T}$ is the extra stress tensor, $\mathbf{v}$ is the velocity vector, and $p$ is the isotropic pressure. We also follow the convention that a tensile stress is negative. ${ }^{18}$

In this paper we use the White-Metzner model:

$$
\mathbf{T}+\lambda(\dot{\gamma}) \stackrel{\mathbf{T}}{\mathbf{T}}=-\eta(\dot{\gamma}) \dot{\gamma}
$$

where $\stackrel{\nabla}{\mathbf{T}}$ denotes the contravariant Oldroyd or upper convected time derivative of the extra stress tensor, $\lambda(\dot{\gamma})$ is a shear ratedependent relaxation time, and $\dot{\gamma}$ is the shear rate defined as $\sqrt{1 / 2 \dot{\gamma}: \dot{\gamma}}$ where $\dot{\gamma}$ is the rate of deformation tensor. For the viscosity function, $\eta(\dot{\gamma})$, we use the Carreau model

$$
\eta=\eta_{0}\left[1+(K \dot{\gamma})^{2}\right]^{(n-1) / 2}
$$

where $\eta, \eta_{0}$, and $K$ are parameters in the model. In simple shear flow the primary normal stress difference, $N_{1}$, is given as:

$$
N_{1}=2 \eta(\dot{\gamma}) \lambda(\dot{\gamma}) \dot{\gamma}^{2}
$$

We then fit the viscometric data of the polystyrene melt which are shown in Figure 1 with an empirical function of the following form for $\lambda(\dot{\gamma})$ :

$$
\lambda(\dot{\gamma})=c_{1}+c_{2} \ln \dot{\gamma}
$$

where $c_{1}$ and $c_{2}$ are constants. In this way $N_{1}$ is exactly predicted by the White-Metzner model in shear flow. We should add that although we can fit the viscometric properties of polystyrene exactly, the White-Metzner (WM) model does not fit the uniaxial 


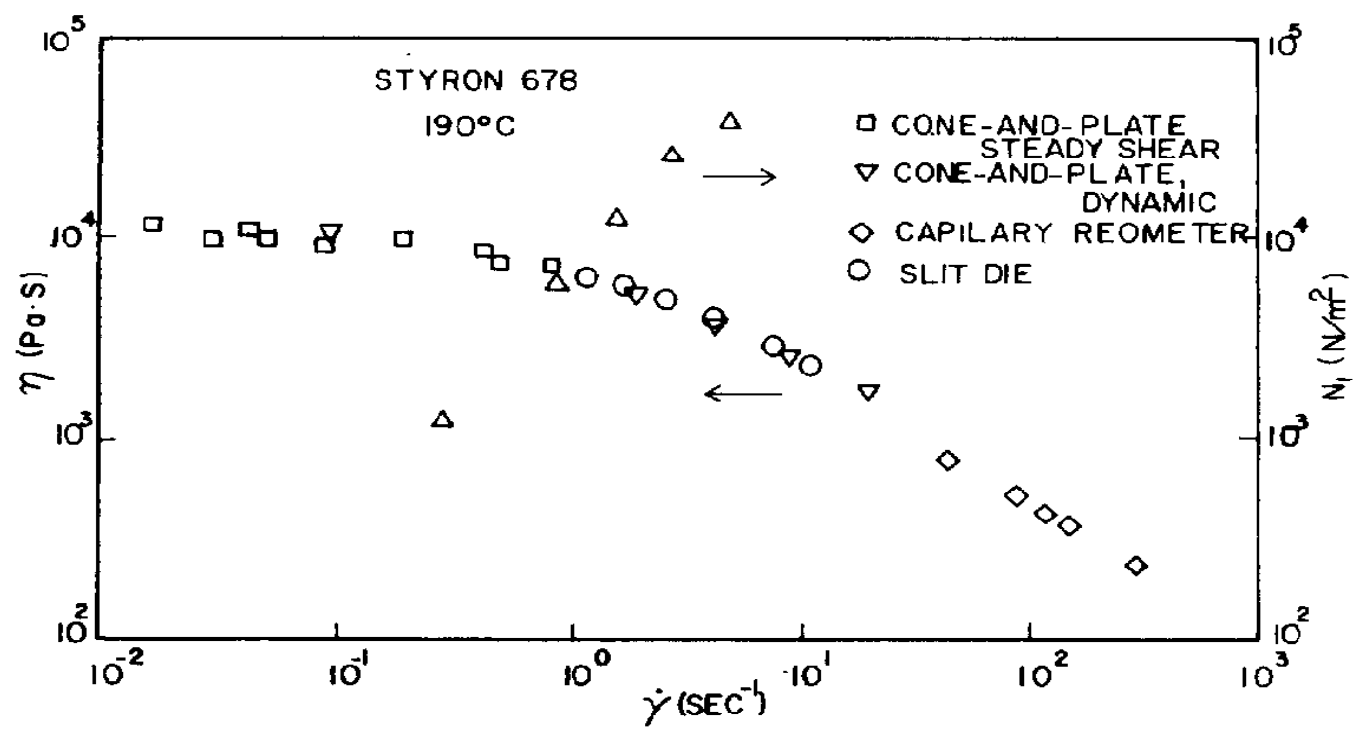

Fig. 1. Viscosity and primary normal stress data for polystyrene(Styron 678) at $190^{\circ} \mathrm{C}$ from ref. 5. ( $\square$ ) Cone-and-plate steady shear; $(\nabla)$ cone-and-plate dynamic; $(\diamond)$ capillary rheometer; $(\bigcirc)$ slit die.

extensional properties of the melt exactly. ${ }^{19}$ In particular, the WM model eventually reaches a critical extension rate where the extensional viscosity, $\bar{\eta}_{e}$, becomes infinite which is contrary to the behavior exhibited by polystyrene. However, because the extension rates are not high throughout the region of interest and because the critical rate is larger than those found here, it should not present a major problem. This will not be true for all flows.

\section{PENALTY-GALERKIN FINITE-ELEMENT METHOD}

The weak form of the equation of motion for the case in which the inertial terms are neglected is: ${ }^{20,21}$

$$
\iint_{\Omega}[\mathbf{T}: \nabla \mathbf{w}+p(\nabla \cdot \mathbf{w})] d \Omega-\oint_{S}(\mathbf{w} \cdot \mathbf{t}) d s=0
$$

where $\mathbf{w}$ is the vector representation of the test functions for $v_{x}$ and $v_{y}$ and $\mathrm{t}$ is the stress (traction) vector acting on the boundaries of the region $\Omega$. The incompressibility condition is treated as a 
constraint on the velocity field which allows us to express the pressure as

$$
p=\gamma_{p} \nabla \cdot v
$$

where $\gamma_{p}$ is the penalty parameter and is taken in this work to be $1 \times 10^{6} * \eta_{o}$, where $\eta_{o}$ is the zero shear rate viscosity. On substitution of Eq. (8) into Eq. (7), we obtain the weak penalty form of the equation of motion as follows:

$$
\iint_{\Omega}\left[\mathbf{T}: \nabla \mathbf{w}+\gamma_{e}(\nabla \cdot v)(\nabla \cdot \mathbf{w})\right] d \Omega-\oint_{S} \mathbf{w} \cdot t d s=0 .
$$

The Galerkin form of the constitutive equation is given as:

$$
\iint_{\mathbf{\Omega}} \mathbf{u} \cdot[\mathbf{T}+\lambda(\dot{\gamma}) \stackrel{\nabla}{\mathbf{T}}+\eta(\dot{\gamma}) \dot{\gamma}] d \mathbf{\Omega}=0,
$$

where $\mathbf{u}$ is a tensorial representation of the test functions for the $x x, x y$, and $y y$ components of $\mathbf{T}$.

The region $\Omega$ is subdivided into rectangles and the velocity and stress fields are represented over each rectangular element by the following polynomial approximations:

$$
\begin{gathered}
v_{i}=\sum_{k} v_{i}^{(k)} \Psi_{k}(x, y), \\
T_{i j}=\sum_{k} T_{i j}^{(k)} \Psi_{k}(x, y),
\end{gathered}
$$

where the $\Psi_{k}$ are taken as bilinear functions of $(x, y)$ and $v_{i}^{(k)}$ and $T_{i j}^{(k)}$ are the nodal values of the components of the velocity and stress fields, respectively. On substituting Eqs. (11) and (12) into Eqs. (9) and (10) and replacing the test functions by $\Psi_{k}$, we obtain the penalty-Galerkin form of the governing equations. The elements of the stiffness matrix are evaluated using four-point quadrature, while reduced integration is used on the penalty terms. The pressure is recovered by means of post computations at the center of each element.

The discretization process just described leads to a system of nonlinear equations of the following form:

$$
\mathbf{S}(X) \cdot X=F .
$$

To solve this system of equations we follow the procedure described by Coleman ${ }^{22}$ which is basically a Picard iteration tech- 
nique. The system of equations in (13) is linearized by evaluating $\mathbf{S}(X)$ at the previous solution, $X^{n}$, and then solving for a new solution, $X^{n+1}$, as indicated below:

$$
\mathbf{S}\left(X^{n}\right) \cdot X^{n+1}=F \text {. }
$$

The iterative scheme given in (14) is used with $\mathbf{S}(X)$ chosen to be independent of the nodal values of $T_{i j}^{(k)}$. In order to accelerate the convergence, $X^{n}$ in (14) is replaced by an extrapolated value, $X_{e}$, where

$$
X_{e}=c X^{n}+(1-c) X^{n-1}
$$

and the optimum value for $c$ was found to be 0.8 . The convergence criterion is

$$
\frac{\left|X^{n+1}-X^{n}\right|}{\left|X^{n+1}\right|} \leq \varepsilon
$$

where $\varepsilon$ is chosen to be $0.01 * \eta_{o}$.

Three meshes are used in the work and are presented as shown in Figure 2. The number of nodes and elements for each mesh are listed in Table I. The main difference between meshes 1 and 2 is the number of elements placed across the mouth of the slot and in the region from the bottom of the slot to the upper wall. The main difference between meshes 2 and 3 is that in mesh 3 more elements are added upstream and downstream of the slot region. In addition, there is more refinement of the mesh near the walls in the undisturbed flow region.

A no-slip velocity boundary condition is used along the solid boundaries while the velocity field at the inlet and outlet positions is assumed to be that of laminar fully developed shear flow. The boundary conditions at the inlet and outlet nodes are established by first solving the problem using the generalized Newtonian fluid model with the boundary conditions that the traction

TABLE I

Convergence Limits for Three Finite-Element Meshes

\begin{tabular}{ccccc}
\hline Mesh & $\begin{array}{c}\text { No. of } \\
\text { Elements }\end{array}$ & $\begin{array}{c}\text { Degrees of } \\
\text { Freedom }\end{array}$ & $\begin{array}{c}\text { Critical Wall Shear } \\
\text { Stress (KPa) }\end{array}$ & $\begin{array}{c}\text { Critical Weissenberg } \\
\text { Number }\end{array}$ \\
\hline 1 & 84 & 535 & 24.0 & 1.02 \\
2 & 256 & 1450 & 14.0 & 0.73 \\
3 & 328 & 1860 & 9.0 & 0.54 \\
\hline
\end{tabular}




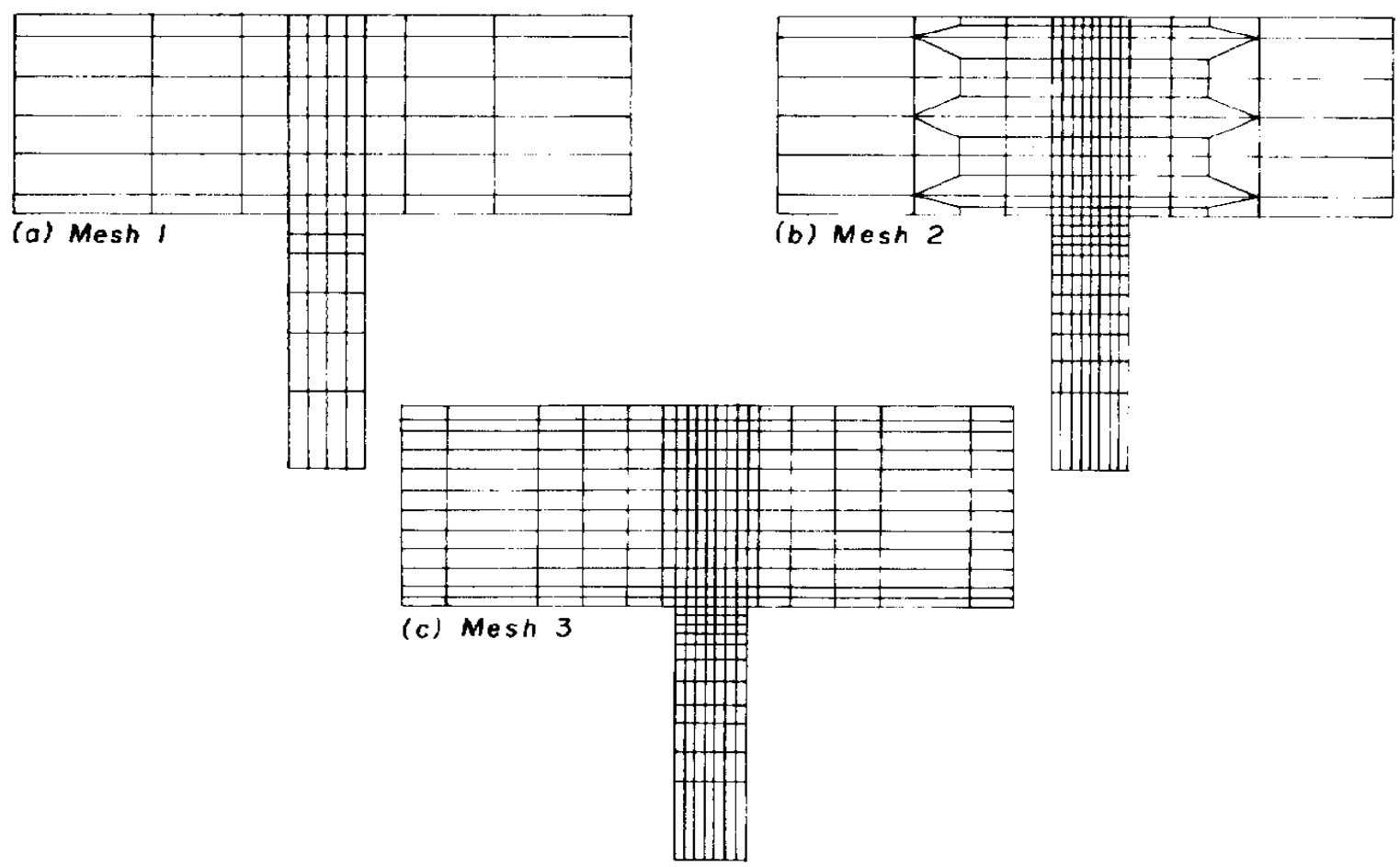

Fig. 2. Finite-element meshes used in this research. The actual dimensions are: slit height $(H)=2.54 \mathrm{~mm}$, the slot width $(B)=0.81 \mathrm{~mm}$, and the slot depth $=3.25 \mathrm{~mm}$.

vector is known along the inlet and outlet elements and there is no-slip of the fluid along the solid boundaries.

\section{COMMENTS ON BIREFRINGENCE RESULTS}

Although details of the birefringence measurements are presented in detail elsewhere ${ }^{5}$ it is important to make a few comments about these measurements. The repeatability of the measurements was of the order of $\pm 5 \%$. The accuracy of these measurements was due in part to the alignment of the optical system which allowed the generation of nearly parallel light rays passing through the flow region. Accurate temperature control of the melt by use of four separately controlled heaters along the die was another key factor in assuring accurate birefringence measurements. By maintaining the melt temperature to within $\pm 0.5^{\circ} \mathrm{C}$, the density variation across the measuring path was minimized. The most difficult part of the measurements was the 
measurement of the principal optic (stress) directions which appear as diffuse black lines through the sample. Only by rotating the analyzer back and forth around the angle desired could the regions of constant principal stresses be identified. Eventually a shear rate was reached where viscous heating as well as side wall effects made the measurements of the isolinics, lines of constant principal stress difference, impossible. It should also be noted that values of the principal directions and principal stresses were extrapolated to various points in space from the measured points by means of linear interpolation.

\section{RESULTS AND DISCUSSION}

The flow region of interest here is shown schematically in Figure 3. There are two regions which we will pay particular attention to and concentrate our comparison between predicted values of the stress field and those measured by means of flow birefringence. These are the centerline of the slot and the slot mouth. The slot centerline is chosen because the validity of the Higashitani and Pritchard theory as discussed by Malkus and Bernstein ${ }^{13}$ and Pike and Baird ${ }^{16}$ depends on the integration of $N_{1} / 2 \sigma$ along this path. This integration path can be rather complex as there is at least one singular point in $N_{1} / 2 \sigma$ near the

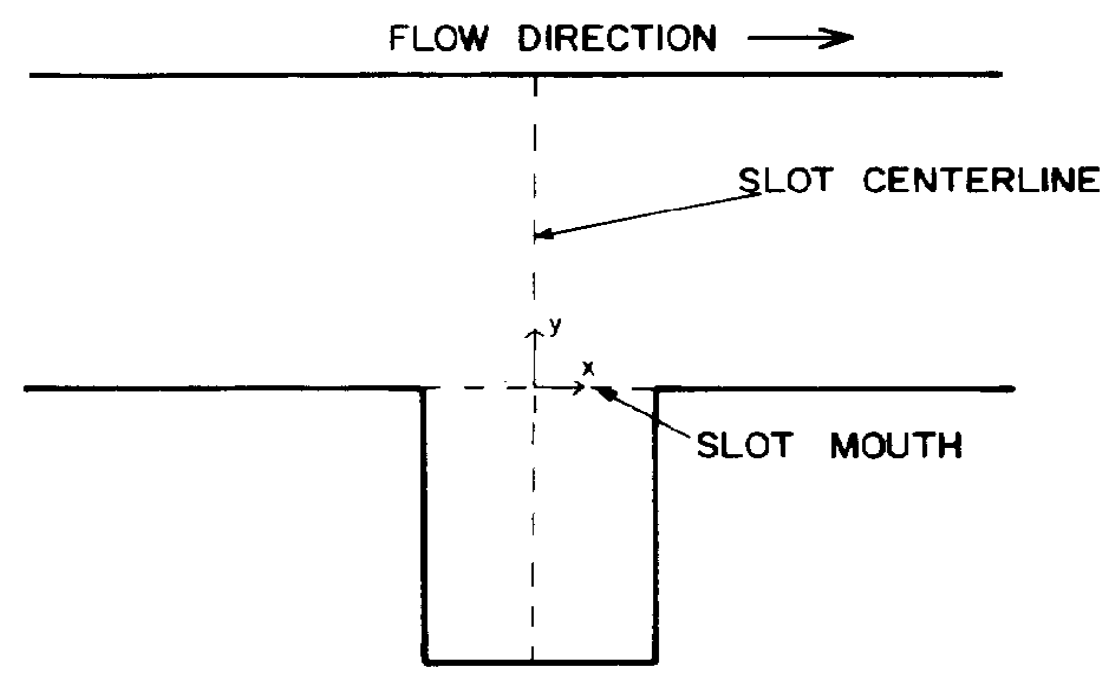

Fig. 3. Schematic of flow over a rectangular slot showing the two regions of interest in this research. 
centerline of the slit. The slot mouth is also an interesting region because it includes two corners and is near the dividing streamline which separates flow in the cavity from the main flow in the die. We first compare stresses predicted by the simulation using the three different meshes with experimental values for conditions where the algorithm is convergent. We then look at results obtained with mesh 1 (coarse mesh) since it gave a convergent solution over a much wider range of values of We and $\sigma_{w}$.

We first compare solutions obtained for the three meshes for conditions where convergent solutions were obtained. Figure 4 shows plotted values of the reduced shear stress versus the reduced $y$-coordinate taken along the centerline of the slot. Here we see there is not only very good agreement between the results obtained using three different meshes, but that all the results agree well with the flow birefringence data. The only place where the agreement is not good is in the region below the mouth of the hole. In this region, for these conditions, the stresses are so low that accurate flow birefringence data is difficult to obtain. Figure 5 presents a similar plot for the reduced normal stress values. Here again we see agreement is quite good between the

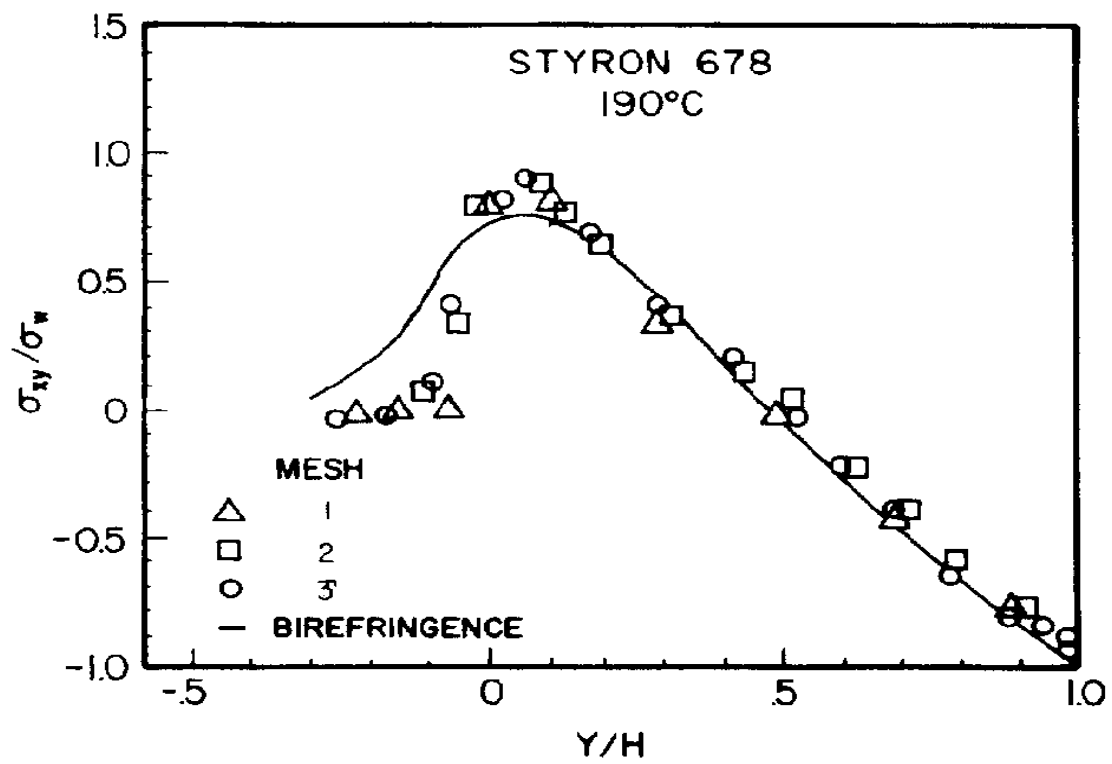

Fig. 4. Reduced shear stress versus the reduced $y$-coordinate along the centerline of the slot at a wall shear stress of $8.93 \mathrm{KPa}$. The solid line is the flow birefringence (-) data taken from ref. 5. Meshes: $\Delta=1 ; \square=2 ; \bigcirc=3$. 


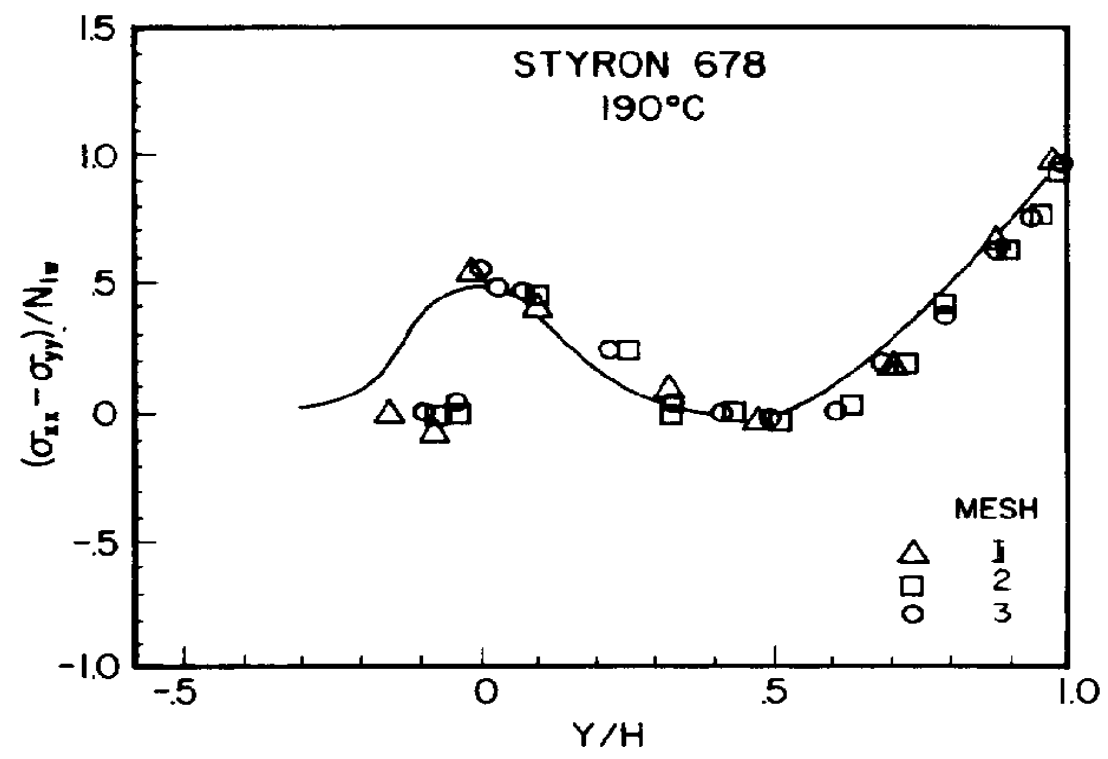

Fig. 5. The normal stress difference reduced with respect to the primary normal stress difference at the wall versus the reduced $y$-coordinate at a wall shear stress of $8.93 \mathrm{KPa}$. The solid line represents the flow birefringence data taken from ref. 5. Meshes: $\triangle=1 ; \Pi=2 ; O=3$.

values obtained using different meshes as well as with the flow birefringence data. In Figures 6 and 7 we compare values of $\sigma /$ $\sigma_{w}$ and the reduced normal stress difference, respectively, versus reduced distance across the mouth of the slot. We see that, in general, values determined using different meshes and those measured by means of flow birefringence are in good agreement. However, for mesh 2 the shear stress values are in poor agreement with the other results. We are uncertain as to the cause of this, but believe it must be related to the elements having angles greater than $90^{\circ}$. Also, at this position, the flow is no longer viscometric as the normal stress difference does not follow the shear stress. Furthermore, at the inlet corner the shear stress actually decreases rather than rises sharply, while there is some significant increase in the normal stress difference. The normal stresses seem to relax over the slot but then rise sharply at the exit plane of the slot. This behavior is observed in both the experimental values and in the simulated values of stress.

Although there was good agreement between the values of stress obtained using the three different meshes, at the next increment of stress (a wall shear stress of $9.0 \mathrm{KPa}$ ), the algorithm 


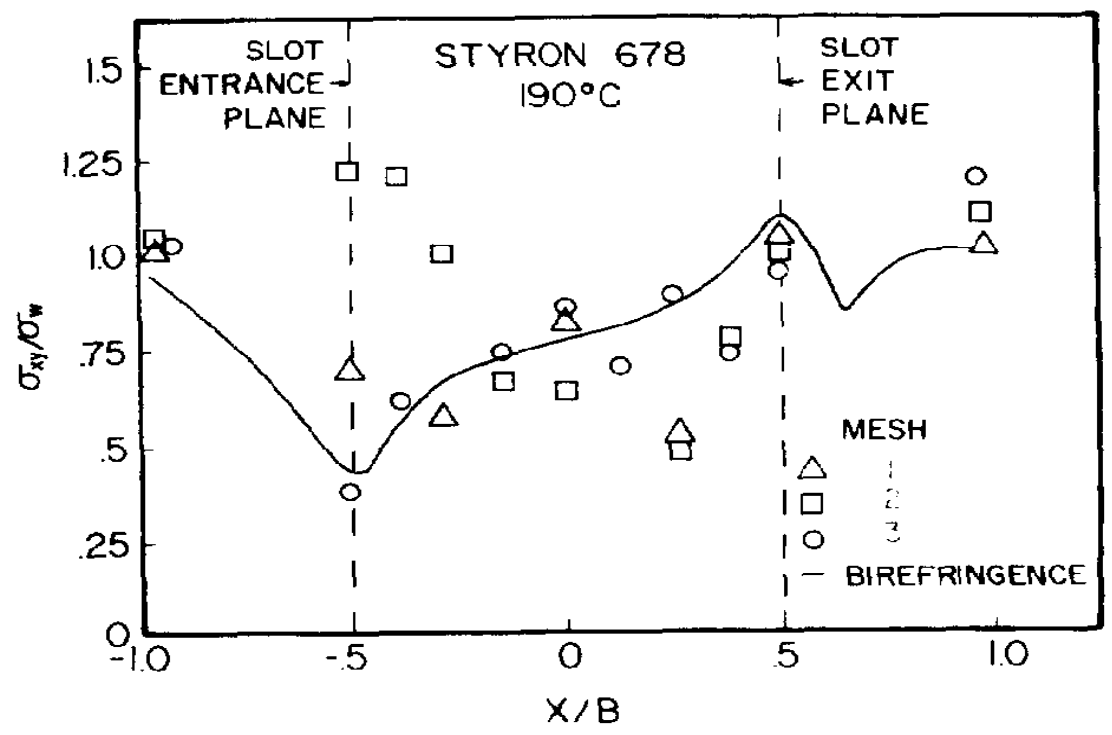

Fig. 6. Reduced shear stress versus the reduced $x$-coordinate across the mouth of the slot at a wall shear stress of $8.93 \mathrm{KPa}$. The solid line represents flow birefringence ( - ) data from ref. 5 . Meshes: $\Delta=1 ; \square=2 ; O=3$.

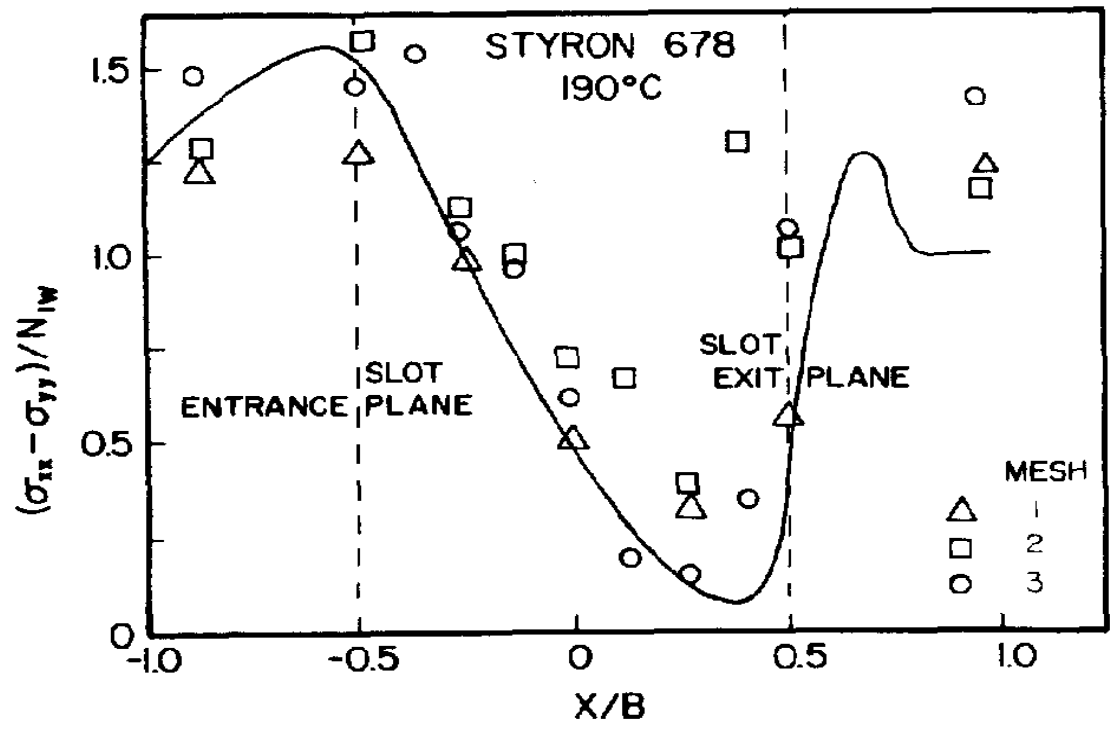

Fig. 7. Reduced normal stress versus the reduced $x$-coordinate across the mouth of the slot at a wall shear stress of $8.93 \mathrm{KPa}$. The solid line represents flow birefringence data from ref. 5 . Meshes: $\Delta=1 ; \square=2$; $\bigcirc=3$. 
would no longer converge for mesh 3 . There was no warning in the stress values in the two regions we studied that convergence difficulties were imminent. A summary of the convergence limits for the three meshes is given in Table I. Here we see that the highest limiting value of We, where we have taken We $=N_{1}, w /$ $2 \sigma_{w}$ where $N_{1}, w$ and $\sigma_{w}$ are values of $N_{1}$ and $\sigma$ evaluated at the wall shear rate, is obtained for the coarse mesh. This behavior has been reported by others for other flow problems. ${ }^{1,23}$ Because it has been reported that coarse meshes used in entry flow simulation can lead to erroneous predictions of vortices, we now look in more detail at the predictions of our simulation using mesh 1 , to see how accurate the predictions are, especially as stress levels increase.

Figures 8 and 9 are plotted values of the reduced shear stress and normal stress difference versus reduced distance across the slot mouth, respectively, for various levels of wall shear stress. Although reduced shear stress values agree well with each other

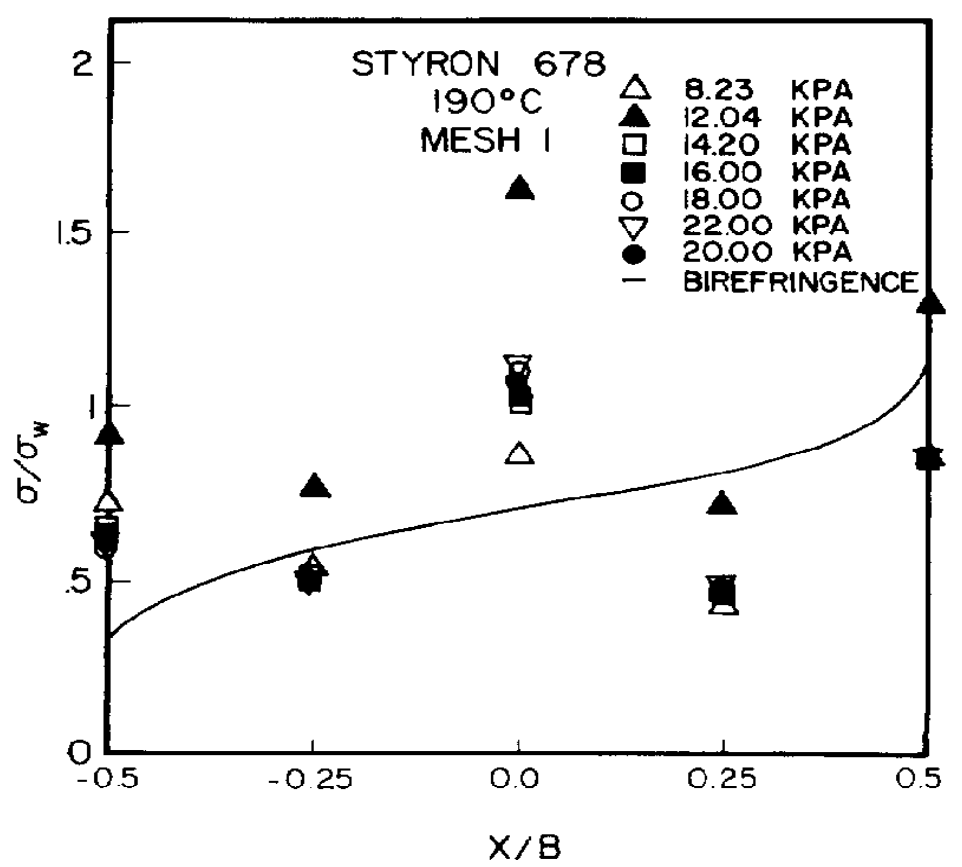

Fig. 8. Reduced shear stress versus the reduced $x$-coordinate across the mouth of the slot for various wall shear stress levels (KPa: $\Delta=8.23 ; \Delta=12.04$; $\square=14.20 ; \square=16.00 ; O=18.00 ; \nabla=22.00 ; \bigcirc=20.00$ ). All calculated values were obtained using mesh 1 . 


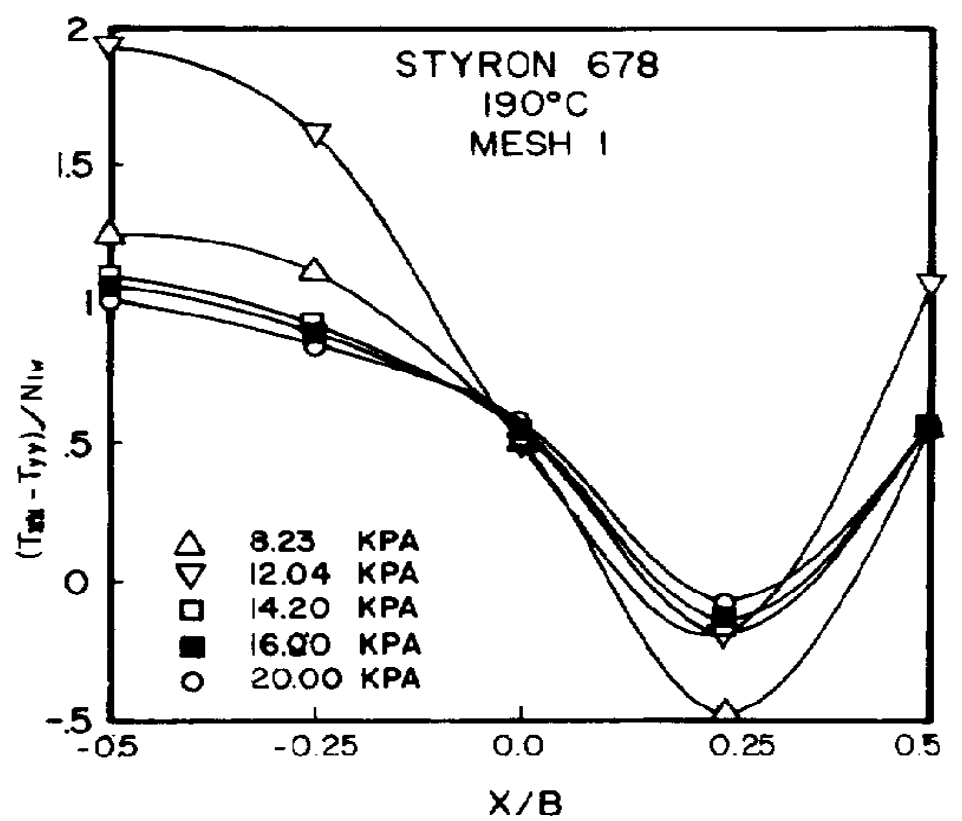

Fig. 9. Reduced normal stress difference versus the reduced $x$-coordinate across the mouth of the slot for various wall shear stress levels (KPa: $\Delta=8.23$; $\nabla=12.04 ; \square=14.20 ; \square=16.00 ; 0=20.00$ ). Mesh 1 was again used to obtain the calculated values.

except in the case of the data obtained at a wall shear stress of $12.04 \mathrm{KPa}$, there is considerable scatter of the predicted values about the flow birefringence data. On the other hand, the normal stress differences agree well with each other and the flow birefringence values. In Figure 10 we have plotted reduced values of $N_{1} / 2 \sigma$ versus reduced shear stress along the centerline of the slot for two different wall shear stress levels. There is good agreement between the predicted values and those measured by means of flow birefringence. Hence, we see that although the mesh is coarse, we still get reasonable agreement between predicted and measured values of stress and that the agreement is still good even as we approach conditions when the numerical algorithm fails to converge.

As a further check of the results obtained using the coarse mesh, we compare values of the hole pressure calculated by taking the difference of (Tyy $+p$ ) at the upper die wall and at the bottom of the slot with values predicted using cone-and-plated data and the Higashitani and Pritchard theory. The values of (Tyy $+p$ ) were evaluated at the centerline rather than by aver- 


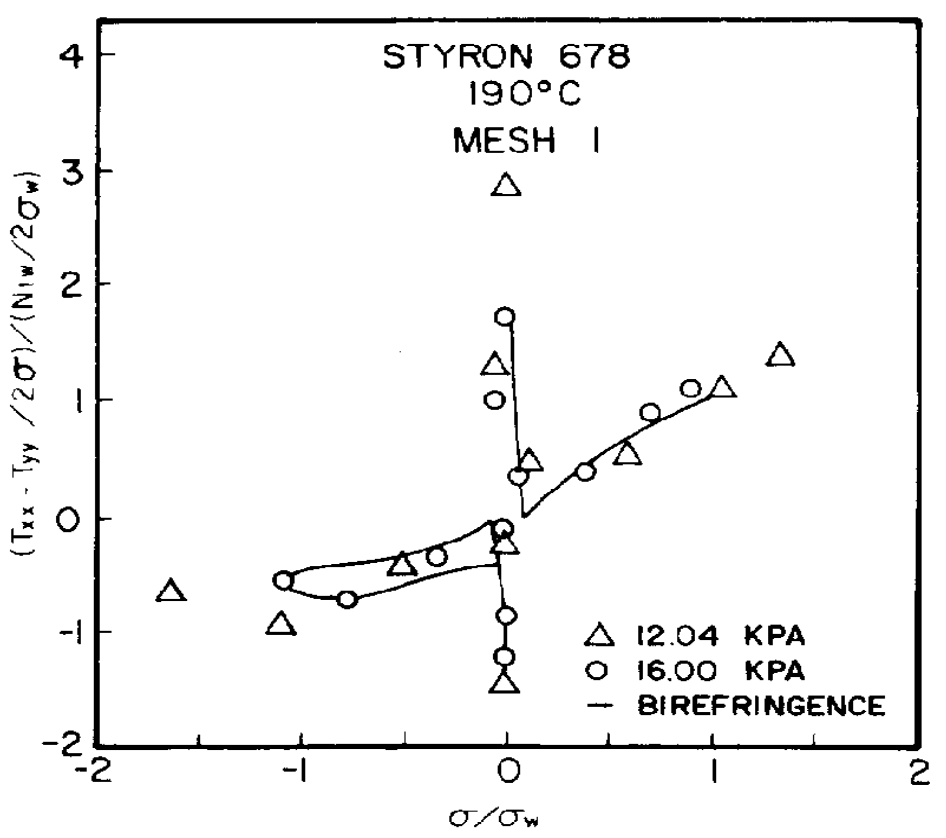

Fig. 10. Reduced ratio of the normal stress to twice the shear stress ratio versus the reduced shear stress along the centerline of the slot. The solid line represents flow birefringence (-) data from ref. 5 . The calculations were carried out at two wall shear stress levels $(\Delta=12.04 ; \bigcirc=16.00)$ using mesh 1 .

aging over the width of the slot. In Figure 11 we observe reasonable agreement between the two sets of values. Again in spite of the coarse mesh we can still get reasonable predictions, and at higher stress levels than are possible with the finer mesh.

As shown in Table I the use of the coarser mesh allowed us to extend the limiting value of We from 0.54 to 1.02 . However, the wall shear stress limit increased by nearly a factor of 3 . Certainly the convergence problem is still a limiting factor in carrying out numerical simulations of viscoelastic fluid flows. We believe part of it is related to the constitutive equation used in the simulation. For example, in the case of the upper convected Maxwell model, We is predicted to increase linearly with increasing shear stress. Hence, in order to reach reasonable values of shear stress, values of We must be of the order of 2 to 3 . However, not many polymeric fluids behave in this manner. To illustrate this point we consider the data presented in Figure 12 in which we have plotted We versus shear rate for polystyrene. Values of $N_{1} / 2 \sigma$ along with $G^{\prime} / G^{*}$, where $G^{\prime}$ is the storage modulus and $G^{*}$ is the complex 


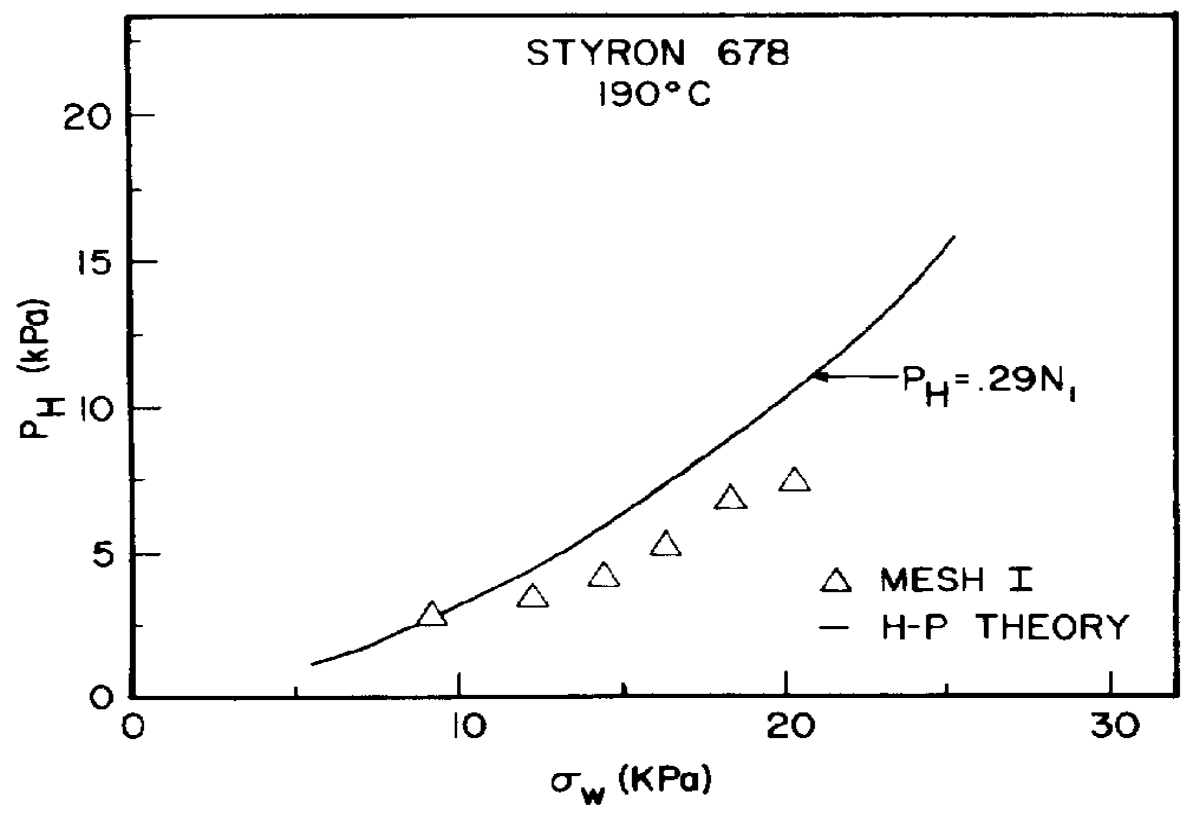

Fig. 11. Calculated values of the hole pressure versus wall shear stress compared with values predicted using the Higashitani and Pritchard (-) theory and cone-and-plate data.

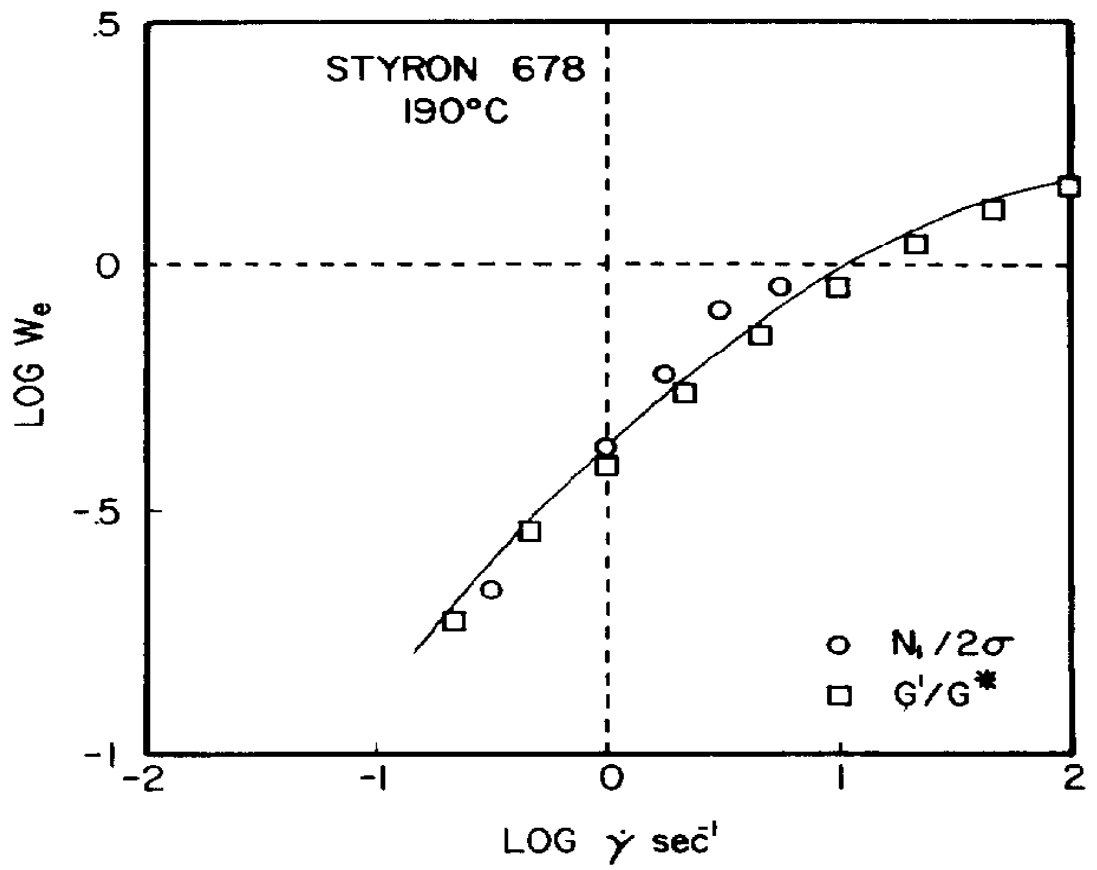

Fig. 12. Weissenberg number versus shear rate for the polystyrene melt used in this study. $\left(O=N_{1} / 2 \sigma, \square=G^{\prime} / G^{*}\right.$.) 
shear modulus, are plotted here. Although the values of $N_{1} / 2 \sigma$ are difficult to obtain in the cone-and-plate apparatus beyond a value of 1.0 , values of $G^{\prime} / G^{*}$ may allow us to look at the behavior of We at higher values. What we observe is that for the polymer used in this work, We approaches an asymptotic value of about 1.5. Furthermore, the constitutive equation used here fits this behavior. Hence, if we reach a value of We of 1.5 , we should be able to carry out calculations up to shear stresses where the onset of melt fracture occurs, which is surely adequate for most polymer processing operations.

\section{CONCLUSIONS}

We have shown here that it is possible to predict stresses which agree quantitatively with values measured by means of flow birefringence when we use a constitutive equation which at least fits the viscometric properties of the melt used in the experiments. We have also found, at least for the case of the hole pressure, that the coarser mesh leads to sufficiently accurate values of stress while permitting us to reach a more reasonable value of elasticity before the numerical algorithm fails to converge. This suggests that there may be an optimum mesh for a given flow problem and constitutive equation. This work also indicates that the elasticity of polymer melts, as determined by the Weissenberg or Deborah numbers, may reach an asymptotic value rather than continue to increase linearly with shear stress. Hence, for engineering applications it may not be necessary to reach values of We much greater than 2.0. The work also points out the importance of coupling experiments on well characterized fluids with the numerical studies.

This work was supported by grants from the Petroleum Research Fund (PRF \#16119-AC7-C) and by the Center for Innovative Technology (Grant \#MAT-85-007).

\section{References}

1. A. R. Davies, S.J. Lee, and M.F. Webster, J. Non-Newtonian Fluid Mech., 16, 117 (1984).

2. K. Walters and M. F. Webster, Phil. Tran. R. Soc. Lond. A, 308, 547 (1982).

3. K. Walters and D. M. Rawlinson, Rheol. Acta, 21, 547 (1982).

4. D. G. Baird, J. Appl. Polym. Sci., 20, 3155 (1976). 
5. R.D. Pike, Ph.D. thesis, Department of Chemical Engineering, Virginia Polytechnic Institute \& State University (1984).

6. T. Cochrane, K. Walters, and M. F. Webster, Phil. Trans. Roy. Soc. London, 301, 163 (1981).

7. A. S. Lodge and L. DeVargas, Rheol. Acta, 22, 151 (1983).

8. M.J. Crochet and M. Bezy, J. Non-Newtonian Fluid Mech., 5, 201 (1979).

9. G. D. Richards and P. Townsend, Rheol. Acta, 20, 261 (1981).

10. P. Townsend, Rheol. Acta, 19, 1 (1980).

11. N. R. Jackson and B.A. Finlayson, J. Non-Newtonian Fluid Mech., 10, 71 (1982).

12. D. G. Baird, Ph.D. thesis, University of Wisconsin-Madison (1974).

13. D.S. Malkus and B. Bernstein, J. Non-Newtonian Fluid Mech., 16, 77 (1984).

14. C. F. Curtiss and R. B. Bird, J. Chem. Phys., 74, 2016 (1981).

15. K. Higashitani and W. G. Pritchard, Trans. Soc. Rheol., 16, 687 (1972).

16. R. D. Pike and D. G. Baird, J. Non-Newtonian Fluid Mech., 16, 211 (1984).

17. D.S. Malkus and M.F. Webster, J. Non-Newtonian Fluid Mech., 25, 93 (1987).

18. R. B. Bird, R.C. Armstrong, and O. Hassager, Dynamics of Polymeric Liquid Fluids, Vol. 1, Fluid Mechanics, Wiley, New York, 1987.

19. S. A. White, Ph.D. thesis, Department of Chemical Engineering, VPI \& SU (1987).

20. J. N. Reddy, An Introduction to the Finite Element Method, McGraw-Hill, New York, 1984.

21. J. N. Reddy, Int. J. Numeric. Meth. Fluids, 2, 151 (1982).

22. C.J. Coleman, J. Non-Newtonian Fluid Mech., 8, 261 (1981).

23. R. Keunings, J. Non-Newtonian Fluid Mech., 20, 11 (1986).

Received November 13, 1987

Accepted February 4, 1988 